\title{
Epidermal growth factor-like domain 8 inhibits the survival and proliferation of mouse thymocytes
}

\author{
FAZLI SUBHAN, TAE-DEUK YOON, HEE JUNG CHOI, IKRAM MUHAMMAD, JIEUN LEE, \\ CHANGWAN HONG, SAE-OCK OH, SUN-YONG BAEK, BONG-SEON KIM and SIK YOON \\ Department of Anatomy, Pusan National University School of Medicine, \\ Yangsan, Gyeongsangnam-do 626-870, Republic of Korea
}

Received April 1, 2013; Accepted July 2, 2013

DOI: $10.3892 /$ ijmm.2013.1448

\begin{abstract}
Thymic epithelial cells (TECs) play a critical role in T-cell development through their intercellular interactions and by producing various soluble proteins, such as growth factors, cytokines and chemokines. In this study, we report a new role for epidermal growth factor-like domain 8 (EGFL8) in the regulation of the survival and proliferation of mouse thymocytes. Mouse recombinant EGFL8 (rEGFL8) protein was produced using an E. coli system and its biological role in mouse thymocytes was determined. The injection of rEGFL8 in mice in vivo resulted in a decrease in the weight of the thymus, as well as in the number of total thymocytes; rEGFL 8 also inhibited thymocyte proliferation and induced thymocyte apoptosis. Furthermore, rEGFL8 suppressed the expression of the Notch downstream targets, Hes1 and Heyl, in mouse thymocytes and TECs, indicating that EGFL8 negatively regulates the Notch signaling pathway in these cells. The identification of the role of EGFL8 in thymocytes may aid in the determination of the fate of thymocytes during T-cell development.
\end{abstract}

\section{Introduction}

Epidermal growth factor-like domain (EGFL), an evolutionarily conserved protein domain, is found in many vertebrate proteins that are involved in several essential cellular activities, such as blood coagulation, fibrinolysis, cell adhesion and development (1). EGFL8, a newly identified member of the EGFL family, was originally identified as a paralog of EGFL7 by a BLAST search of the mouse genome (2). EGFL7 has recently emerged as a secreted angiogenic signaling molecule,

Correspondence to: Professor Sik Yoon, Department of Anatomy, Pusan National University School of Medicine, 49 Busandaehak-ro, Mulgeum-eup, Yangsan, Gyeongsangnam-do 626-870, Republic of Korea

E-mail: sikyoon@pusan.ac.kr

Key words: epidermal growth factor-like domain 8, thymocyte, T-cell development, Hes1, Hey1 which enhances vasculogenesis and angiogenesis by promoting endothelial cell adhesion, proliferation, chemoattraction, migration, sprouting and invasion (3-7). Furthermore, EGFL7 stimulates embryonic stem cell proliferation, but inhibits the proliferation of adult neuronal stem cells, demonstrating its differential mechanisms of action during cell proliferation between different stem cell types $(7,8)$.

However, little is known about the characterization and the biological role of EGFL8. A structural analysis of the EGFL8 protein predicted that it is a secretory protein (9). EGF-like repeats, located in the extracellular domains of EGFL8 and Notch receptors, play a central role in controlling the Notch signaling pathway (10). As recently demonstrated, EGFL8 expression was significantly decreased in patients with colorectal and gastric cancer, suggesting that EGFL8 may have a distinct expression pattern and mechanism of action in cancer progression $(11,12)$.

We have previously demonstated that the mouse EGFL8 gene plays a functional role in T-cell development in a gainof-function and loss-of-function study with an EGFL8 gene overexpressing vector and EGFL8 siRNA (13). Based on these previous findings, in the present study, we investigated the functional role of the EGFL8 protein in mouse thymocytes and thymic epithelial cells (TECs), which are pivotal for both T-cell development and T-cell repertoire selection (14). To identify the potential importance of the EGFL8 protein, in this study, we designed and optimized a protocol to produce and purify large amounts of mouse recombinant EGFL8 (rEGFL8) protein. Using high-purity mouse rEGFL8, we demonstrate that EGFL8 inhibits the survival and proliferation of thymocytes during T-cell development. In addition, the mechanistic regulatory role of EGFL8 in mouse thymocytes and TECs was determined.

\section{Materials and methods}

Cell line and cell culture. The mouse thymic cortical epithelial reticular cells (1308.1) were kindly provided by Dr Barbara B. Knowles (The Jackson Laboratory) (15). The cells were cultured in Dulbecco's modified Eagle's medium (DMEM), containing $10 \%(\mathrm{v} / \mathrm{v})$ fetal bovine serum (FBS), $100 \mathrm{U} / \mathrm{ml}$ penicillin and $100 \mu \mathrm{g} / \mathrm{ml}$ streptomycin (all from Gibco Life Technologies, Grand Island, NY, USA) at $37^{\circ} \mathrm{C}$ in $5 \% \mathrm{CO}_{2}$ incubator. 
Cloning of mouse EGFL8 gene. The coding sequences of mouse EGFL8 total cDNA were isolated and amplified by PCR and cloned into pcDNA3.1 (Life Technologies, Carlsbad, CA, USA). They were amplified by PCR using the following oligonucleotide primers: EGFL8 forward, 5'-TTT CAA AGA GAG TTT GGG AGT G-3' and reverse, 5'-CAC CAC GTG T'T CTG TGG TA-3' to create the Ncol and Xhol restriction sites at the start and stop codon sites. The PCR product was cloned into the pET28a vector, which carries a C-terminal His.Tag/ thrombin/T7·Tag configuration plus an optional C-terminal His.Tag sequence.

Expression of mouse EGFL8 gene. The E. coli bacteria were cultured for $2 \mathrm{~h}$, stimulated by the addition of $0.2 \mathrm{mM}$ IPTG, and then cultured for an additional $4 \mathrm{~h}$. The bacteria were harvested immediately by centrifugation at $6,000 \mathrm{rpm}$ for $8-10 \mathrm{~min}$, and the pellet was either frozen at $-80^{\circ} \mathrm{C}$ until purification or directly resuspended in $60 \mathrm{ml}$ lysis buffer [50 mM Tris- $\mathrm{HCl}$ (pH 8.0), $100 \mathrm{mM} \mathrm{NaCl}$ and $5 \mathrm{mM}$ EDTA]. Subsequently, $0.5 \%$ Triton X-100, $0.1 \mathrm{mM}$ phenylmethylsulfonyl fluoride (PMSF) and $1 \mathrm{mM}$ dithiothreitol (DTT) were added to this pellet. Subsequently, the pellet was sonicated and centrifuged at 12,000 $\mathrm{rpm}$ for $15 \mathrm{~min}$. The supernatant was discarded and the pellet was resuspended in lysis buffer. The following steps were repeated as above except for the addition of $10 \mathrm{mM} \mathrm{MgCl}, 0.01 \mathrm{mg} / \mathrm{ml} \mathrm{DNase}$ and $0.1 \mathrm{mg} / \mathrm{ml}$ lysozyme to the Triton X-100, PMSF and DTT mixture. The pellet mixture was incubated for $20 \mathrm{~min}$ at room temperature, sonicated and centrifuged as described above. The supernatant was discarded and the pellet was resuspended in $60 \mathrm{ml}$ lysis buffer. Subsequently, only PMSF and DTT were added, sonicated and centrifuged as described above. The supernatant was discarded, and the pellet was resuspended in $40 \mathrm{ml}$ of $8 \mathrm{M}$ urea [100 mM Tris- $\mathrm{HCl}$ (pH 8.0), $50 \mathrm{mM}$ glycine]. The pellet was then slowly shaken for $1 \mathrm{~h}$ at room temperature to completely solubilize the proteins. After a short centrifugation to remove non-solubilized proteins, the protein concentration was determined by a NanoDrop 2000 spectrophotometer (Thermo Scientific, Wilmington, DE, USA). The proteins $(1-2 \mathrm{mg} / \mathrm{ml})$ were dialyzed through a dialysis bag. Two liters of dialysis buffers [20 mM Tris- $\mathrm{HCl}$ (pH 8.0), $150 \mathrm{mM} \mathrm{NaCl}$ and $0.1 \mathrm{mM}$ DTT] were used at $4^{\circ} \mathrm{C}$ for 12 to $15 \mathrm{~h}$ and the process was repeated. The third dialysis buffer was used under the same conditions except for the absence of DTT.

Ni-NTA column purification. Purification of His-tagged proteins for the recombinant protein was then performed. The column was washed with distilled water and then with $6 \mathrm{X}$ Ni-NTA washing buffer. The protein mixture was added to the Ni-NTA column and slowly passed through the column. The bound EGFL8 protein was eluted with 6X Ni-NTA elution buffer. The eluted protein was subjected to sodium dodecyl sulfate-polyacrylamide gel electrophoresis (SDS-PAGE) after determining its concentration.

Mass spectrometry. The rEGFL8 protein was subjected to $10 \%$ SDS-PAGE, stained with Coomassie blue, and the protein bands were excised from the gel. The excised gel pieces were transferred to microcentrifuge tubes containing $0.5 \mathrm{ml}$ of distilled water. The protein was then digested with trypsin. The single band was used for matrix-assisted laser desorption/ionization time-of-flight mass spectrometry (MALDI-TOF-MS/MS).

Experimental animals and treatment with rEGFL8 in vivo. C57BL/6 mice (Dae Han Bio Link, Chungbuk, Korea) were intravenously (i.v.) injected with rEGFL-8 (100 $\mu \mathrm{g})$ and were sacrificed 12 h, 1, 2 and 3 days after injection. Animal care and all experimental procedures were conducted in accordance with the 'Guide for Animal Experiments' published by the Korean Academy of Medical Sciences.

Proliferation and apoptosis assay. The mice were injected intraperitonealy (i.p.) with BrdU (BD Biosciences Pharmingen, San Diego, CA, USA) or with PBS. The thymus was isolated by dissection $2 \mathrm{~h}$ after BrdU injection. The isolated cells were stained for CD4, CD8, CD25, CD44 and BrdU according to the BrdU Flow kit manual (BD Biosciences Pharmingen). For the apoptosis assay, the isolated cells were stained for CD4, CD8, CD25, CD44 and Annexin V according to the Annexin V detection kit protocol (BD Biosciences Pharmingen).

Flow cytometric analysis. The following fluorochromeconjugated monoclonal antibodies (mAbs) were purchased from BD Biosciences Pharmingen: Pacific Blue-conjugated anti-CD4 (RM4-5), allophycocyanin (APC)-Cy7-conjugated anti-CD8 (53-6.7), PE-labeled anti-CD25 (PC61) and APC antiCD44 (IM7). Flow cytometric analysis was performed using a FACSCanto II flow cytometer (BD Biosciences Pharmingen), and the acquired data were analyzed using the FlowJo software (Tree Star, Ashland, OR, USA).

Electron microscopy. For electron microscopic investigation, all specimens were processed according to a standard procedure. The ultra-thin sections were examined under a JEOL-1200 EXII transmission electron microscope.

Western blot analysis. The TECs were incubated with $100 \mathrm{ng} / \mathrm{ml}$ rEGFL 8 for $12 \mathrm{~h}$. The thymocytes were isolated from the mice injected with $100 \mu \mathrm{g}$ of rEGFL8 i.v., after $12 \mathrm{~h}, 1$ and 2 days. Following treatment with rEGFL8, the thymocytes and TECs were washed with cold PBS and the total proteins were then extracted from the cultured cells using a protein extraction solution (iNtRON Biotechnology, Seongnam, Korea) supplemented with a protease inhibitor mixture (Sigma-Aldrich, St. Louis, MO, USA). Protein concentrations were measured using the Bradford protein assay kit (Bio-Rad, Hercules, CA, USA). Anti-Hes-1 (sc-166378), anti-Hey-1 (sc-28746) (both from Santa Cruz Biotechnology, Santa Cruz, CA, USA) and anti- $\beta$-actin (Abcam, Cambridge, UK) antibodies were used for immunoblot analysis. The goat anti-mouse IgG-HRP (7076) and goat anti-rabbit IgG-HRP (7074) (both from Cell Signaling Technology, Danvers, MA, USA) were used as the secondary antibodies. Immunoreactivity was detected and quantified using a LAS-3000 imaging system (Fujifilm, Tokyo, Japan).

Statistical analysis. Data are expressed as the means \pm SD. Statistical analyses were performed using the Student's t-test. A value of $\mathrm{P}<0.05$ was considered to indicate a statistically significant difference. 
A

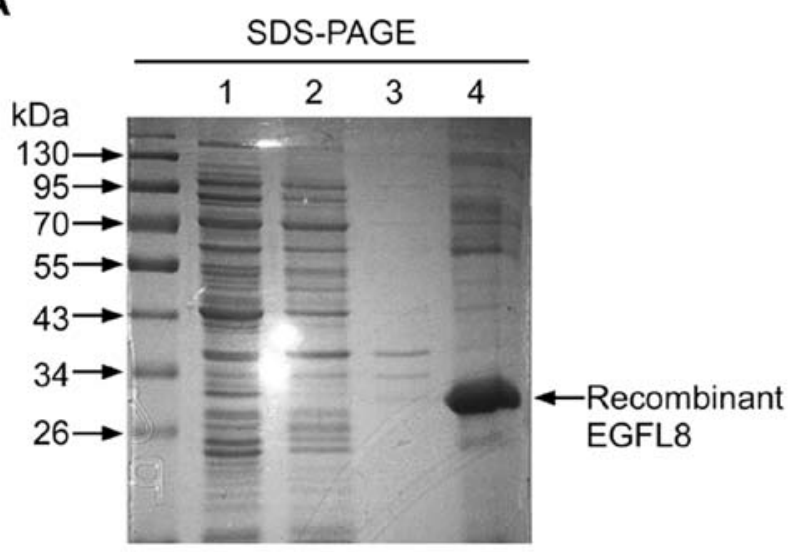

B

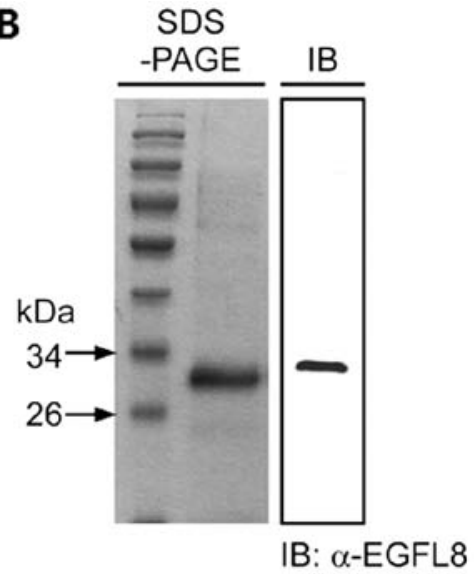

Figure 1. (A) The mouse recombinant epidermal growth factor-like domain 8 (rEGFL8) produced in E. coli and stained with Coomassie blue. Lane 1, first supernatant; lane 2, second supernatant; lane 3, third supernatant; and lane 4, protein pellet. (B) Ni-NTA column purification and western blot analysis of rEGFL8. SDS-PAGE and Coomassie blue staining showed no detectable impurities after Ni-NTA column purification. Immunoblot (IB) analysis demonstrated a single, specific band of rEGFL8.

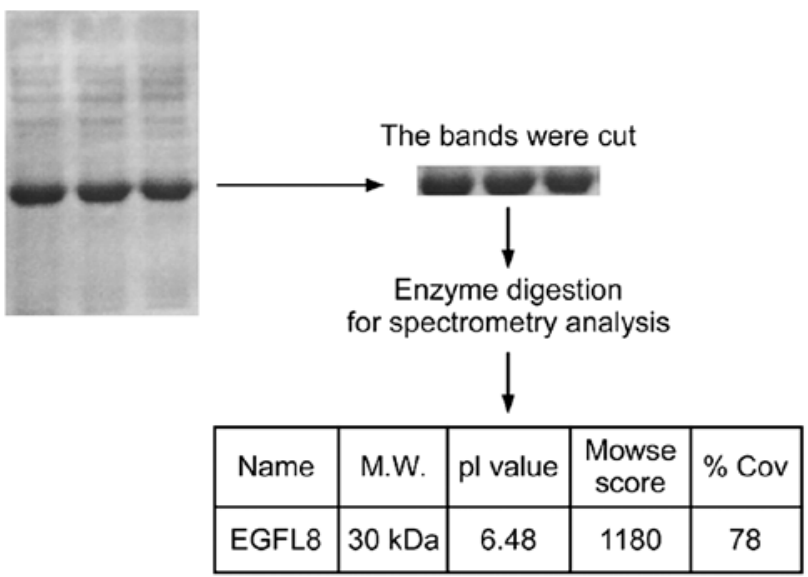

Figure 2. Recombinant epidermal growth factor-like domain 8 (rEGFL8) protein bands were cut for spectrometry analysis and trypsinized. Mass spectrometric analysis of rEGFL8 determined the molecular weight (M.W.), PI value, Mowse score and the percentage coverage (\% Cov) of rEGFL 8 .

\section{Results}

Expression and purification of mouse rEGFL8 protein.rEGFL8 was expressed in E. coli and purified. The harvested rEGFL8 protein was stained with Coomassie blue in $10 \%$ SDS-PAGE (Fig. 1A). We produced high-purity proteins after the third lysis step, suggesting that most of the secreted fusion proteins and most of the other bacterial proteins were removed by the lysis procedure. The dialysis and refolding steps were performed in the presence of urea, mainly as EGFL8 is prone to precipitation at high concentrations and is also prone to protein interactions with the exchange groups on the columns. However, to recover the biological activity of rEGFL8, urea needs to be removed and the protein should be refolded properly. The dialysis protein was analyzed on SDS-PAGE in comparison with cell lysates and supernatants to confirm the successful recovery of the recombinant protein after solubilization with $8 \mathrm{M}$ urea. Fig. 1A illustrates an exact size of the desired protein for this study with no contaminated product from the $E$. coli cells. This result also verified that most or all of the inclusion bodies of
rEGFL8 protein were solubilized in $8 \mathrm{M}$ urea. The Ni-NTApurified protein was loaded onto $10 \%$ SDS-PAGE for total protein analysis (Fig. 1B). The total Ni-NTA column-purified protein showed a single protein band with a specific size, which confirmed the successful rEGFL8 protein purification through the Ni-NTA column. The Ni-NTA-purified protein was also analyzed by western blot analysis for further confirmation (Fig. 1B). The western blot analysis result also showed a single and specific band of rEGFL8 protein. For further characterization, the rEGFL8 protein was run on 10\% SDS-PAGE and the band was cut and trypsinized before loading onto the spectrometer (Fig. 2). All liquid chromatography (LC)-MS/MS data were submitted to a Mascot search; the peaks from the purified rEGFL8 protein sample are shown in Fig. 3, with the masses indicated on the top of each peak. The identified molecular weight of purified rEGFL8 measured by MS was $30.09 \mathrm{kDa}$, which was almost identical to the theoretical value.

Negative effects of $r E G F L 8$ on the weight of the thymus and the number of thymocytes. To investigate the biological activity of rEGFL8 protein in mouse thymocytes, $100 \mu \mathrm{g}$ of rEGFL8 protein were injected i.v. into the mice, and the weight of the thymus and the total number of thymocytes were determined (Fig. 4). The weight of the thymus and the total number of thymocytes were significantly decreased after the rEGFL8 injection. The weight of the thymus and the number of thymocytes diminished from 1 day after rEGFL8 injection, and these effects reached a peak value at 3 days. The observation that the number of thymocytes markedly decreased after the administration of rEGFL8 raised the question of whether the subsets of thymocytes were differentially affected by treatment with rEGFL8. To address this issue, mouse thymocytes were freshly isolated 1,2 and 3 days after rEGFL8 injection, and were stained with anti-CD4 and anti-CD8 mAbs (Fig. 5). Of note, there was a drastic decrease in both the percentage and number of $\mathrm{CD} 4^{+} \mathrm{CD}^{+}$double-positive (DP) thymocytes 3 days after rEGFL8 injection (Figs. 5 and 6). Moreover, the $\mathrm{CD}^{+}$single-positive (SP) and $\mathrm{CD} 8^{+} \mathrm{SP}$ thymocyte subsets were also significantly reduced in number compared with the wild-type subset, although to a lesser degree than the $\mathrm{CD}^{4}{ }^{+} \mathrm{CD} 8^{+}$DP thymocytes on day 3 (Fig. 6). 


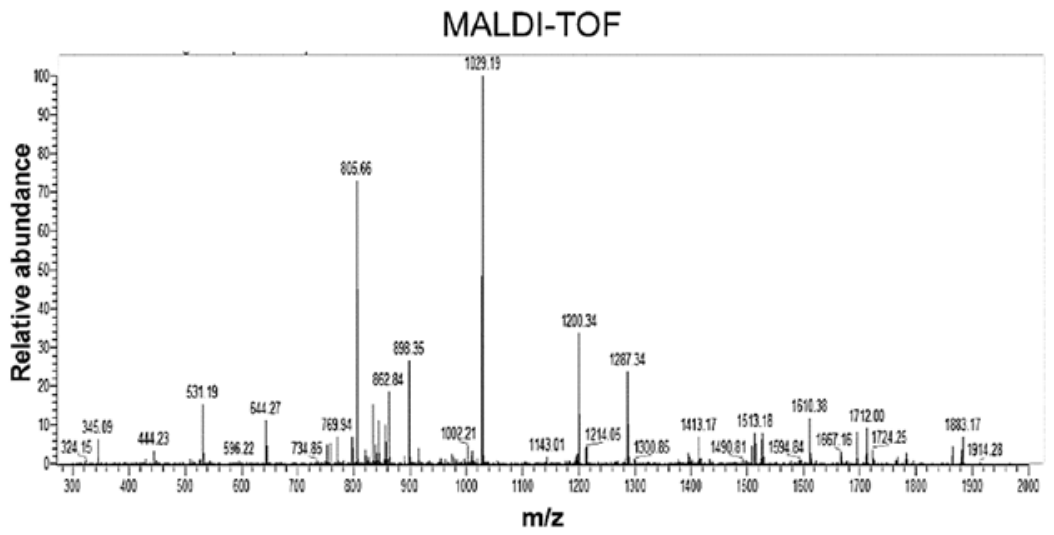

Figure 3. MALDI-TOF MS analysis of the protein band that was excised from the SDS-PAGE gel and in-gel digestion using trypsin. The mass peaks are shown. Numbers on the top of each peak indicate the mass.
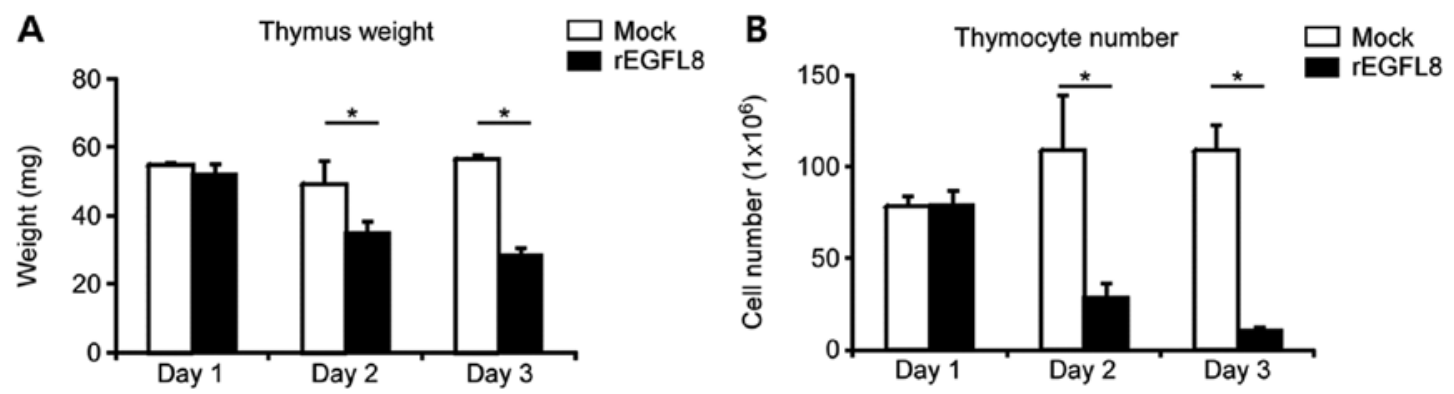

Figure 4. (A) The weight of the thymus and (B) the number of thymocytes following the injection of recombinant epidermal growth factor-like domain 8 (rEGFL8). The weight of the thymus and the number of thymocytes were determined 1,2 and 3 days after rEGFL 8 injection. The weight of thymus and the number of thymocytes were significantly decreased compared with the control mice. Data are the means + SD of at least 3 independent experiments with $\geq 4$ animals per group. ${ }^{*}<<0.05$ compared with the corresponding control value as determined by the Student's t-test.

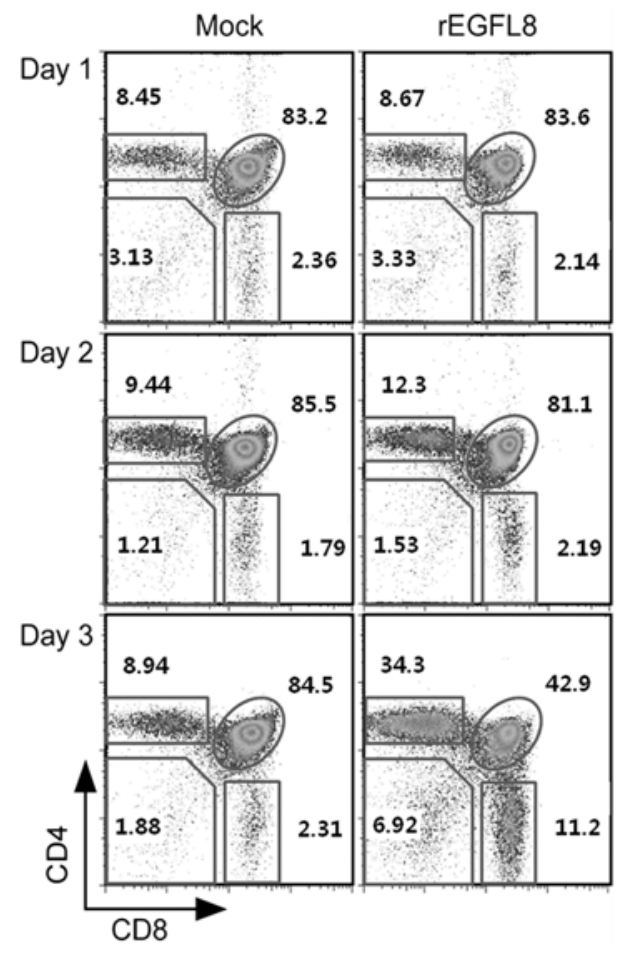

Figure 5. Flow cytometric analysis of thymocyte subsets 1,2 and 3 days after the injection of recombinant epidermal growth factor-like domain 8 (rEGFL8) in vivo. Thymocytes were stained with monoclonal anti-CD4 and anti-CD8 antibodies. Dot blots show CD4/CD8 thymocyte profiles at the indicated time points.

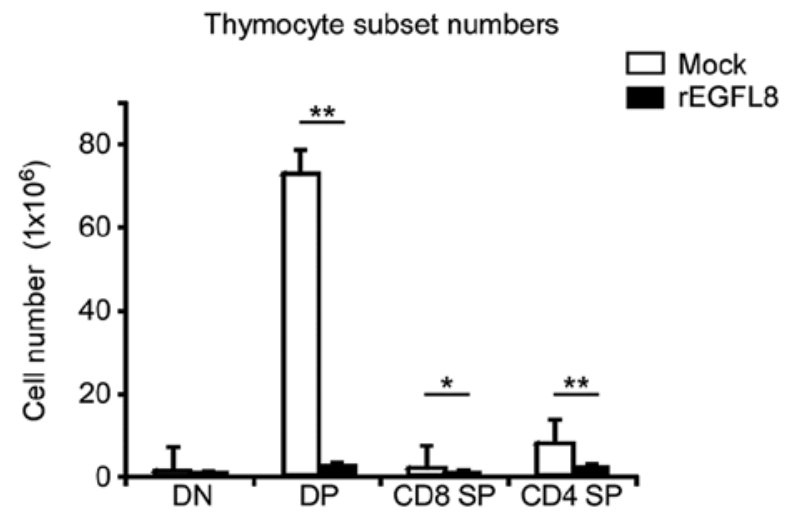

Figure 6. Analysis of the number of thymocytes 3 days after in vivo injection with recombinant epidermal growth factor-like domain 8 (rEGFL8), Thymocyte numbers were determined in each subset of thymocytes (DN, DP, CD8 SP and CD4 SP) after rEGFL8 protein injection. EGFL8 regulates mouse T-cell development in vivo. Data are representative of 3 independent experiments with $\geq 4$ animals per group (means $\pm \mathrm{SD}$ ). ${ }^{*} \mathrm{P}<0.05$ and ${ }^{* * *} \mathrm{P}<0.01$ $\mathrm{DN}$, double-negative; DP, double-positive; SP, single-positive.

Inhibitory effect of $r E G F L 8$ on the survival and proliferation of thymocytes. The reduction in the number of thymocytes follwing treatment with rEGFL8 also raised the question of whether these changes occur due to the inhibition of cell proliferation or the promotion of apoptosis of thymocytes by rEGFL8. To shed light on this matter, the effect of rEGFL8 on 
A

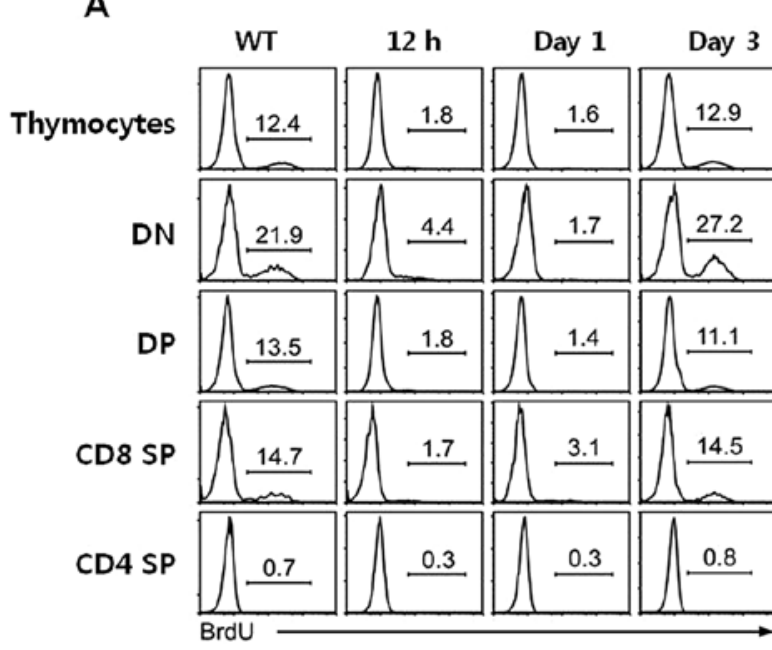

B

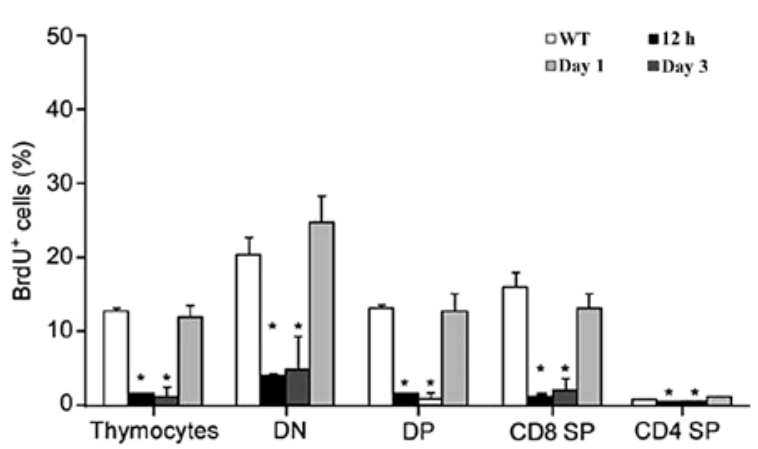

Figure 7. (A) The effect of epidermal growth factor-like domain 8 (EGFL8) on cell proliferation was assessed by a BrdU incorporation assay and subsequent FACS analysis. Mice were injected intraperitonealy (i.p.) with BrdU $12 \mathrm{~h}, 1$ and 3 days following treatment with recombinant EGFL8 protein (rEGFL8) and sacrificed $2 \mathrm{~h}$ later. Thymocytes were stained with anti-CD4, anti-CD8, anti-CD25, anti-CD44 and anti-BrdU mAbs. The histogram shows BrdU+ thymocytes gated on the 4 major subsets of thymocytes (DN, DP, CD8 SP and CD4 SP) of rEGFL8-treated mice at the indicated time points. (B) The bar graph is a summary of the BrdU+ thymocyte frequency. All major thymocyte subsets, particularly at $12 \mathrm{~h}$ and 1 day after EGFL8 injection, exhibited a decrease in the percentage of $\mathrm{BrdU}^{+}$cells compared with the control mice. Groups of mice were analyzed at each time point and data are presented as the means $\pm \mathrm{SD}$. " $\mathrm{P}<0.05$. DN, double-negative; DP, double-positive; SP, single-positive.

A

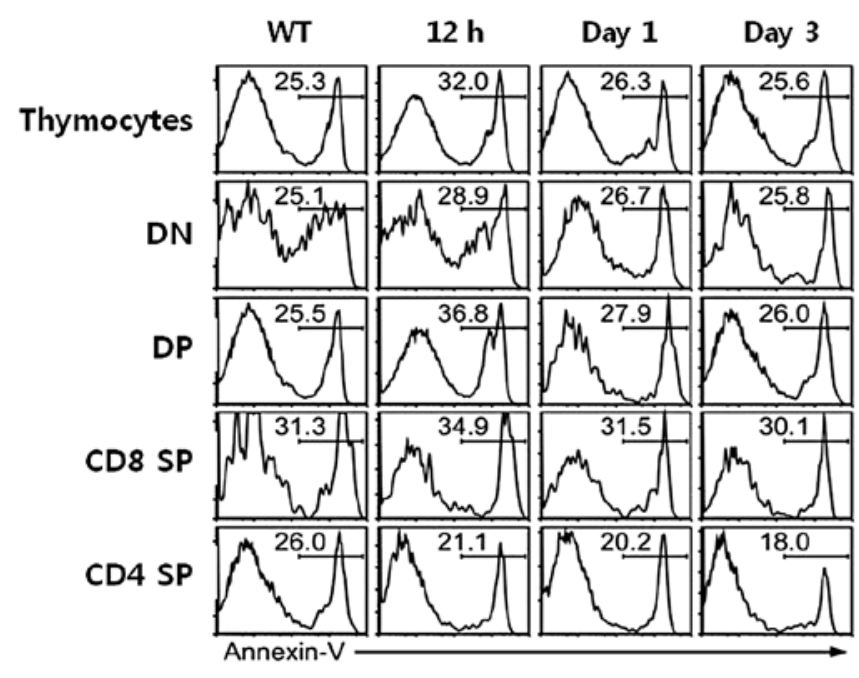

B

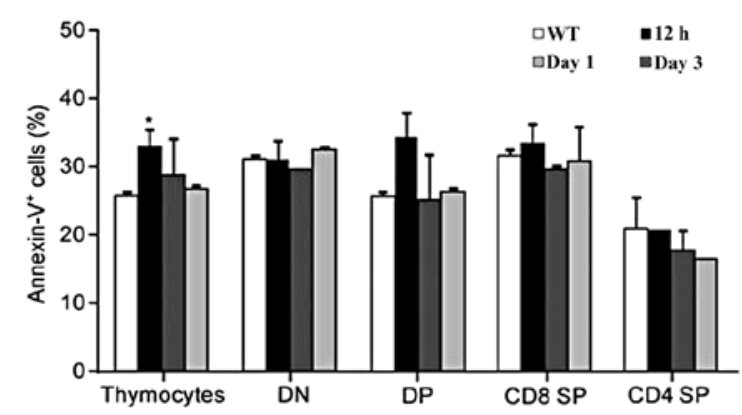

Figure 8. (A) The effect of epidermal growth factor-like domain 8 (EGFL8) on cell survival was assessed by FACS analysis upon the administration of recombinant EGFL8 (rEGFL8) protein for $12 \mathrm{~h}, 1$ and 3 days. Thymocytes from rEGFL8- or mock-treated mice were stained with the FITC-Annexin V and PI reagents. The histogram shows Annexin $\mathrm{V}^{+}$thymocytes in the indicated thymocyte subpopulation. (B) The bar graph is a summary of the Annexin $\mathrm{V}^{+}$ thymocyte frequency. The percentage of apoptosis in the mouse thymocytes $12 \mathrm{~h}$ after EGFL8 injection significantly increased compared with the control thymocytes. Groups of mice were analyzed at each time point and data are presented as the means $\pm \mathrm{SD}$. " $\mathrm{P}<0.05$.

cell proliferation was first investigated $12 \mathrm{~h}, 1$ and 3 days after rEGFL8 injection. Freshly isolated thymocytes from the thymus $2 \mathrm{~h}$ after BrdU injection were stained for anti-CD4, anti-CD8, anti-CD25, anti-CD44 and anti-BrdU, and subsequently flow cytometric analysis was performed (Fig. 7). Remarkably, the $\mathrm{BrdU}^{+}$thymocyte numbers were robustly reduced in the $\mathrm{DN}$, $\mathrm{DP}, \mathrm{CD} 4^{+} \mathrm{SP}$ and $\mathrm{CD} 8^{+} \mathrm{SP}$ thymocyte subsets $12 \mathrm{~h}$ and 1 day after rEGFL8 injection, indicating that rEGFL8 profoundly inhibited the cell proliferation of all 4 major thymocyte subsets. The number of $\mathrm{BrdU}^{+}$thymocytes returned to a level similar to that of the normal control mice at 3 days post-treatment with rEGFL8 (Fig. 7). Subsequently, to determine whether rEGFL8 induces apoptosis during thymocyte development, thymocytes were stained with Annexin V antibody, as well as anti-CD4 and anti-CD8 mAbs after rEGFL8 injection for $12 \mathrm{~h}, 1$ and 3 days. A relatively low but significant level of apoptosis was induced in total thymocytes $12 \mathrm{~h}$ after rEGFL8 injection (Fig. 8). The number of Annexin $\mathrm{V}^{+}$cells reached peak values $12 \mathrm{~h}$ following treatment with rEGFL8 (Fig. 8). These results clearly indicate that EGFL8 is not a potent inducer of apoptosis in mouse thymocytes.

To determine whether EGFL8 causes morphological changes in the mouse thymus at the ultrastructural level, transmission electron microscopy was performed after the injection of 

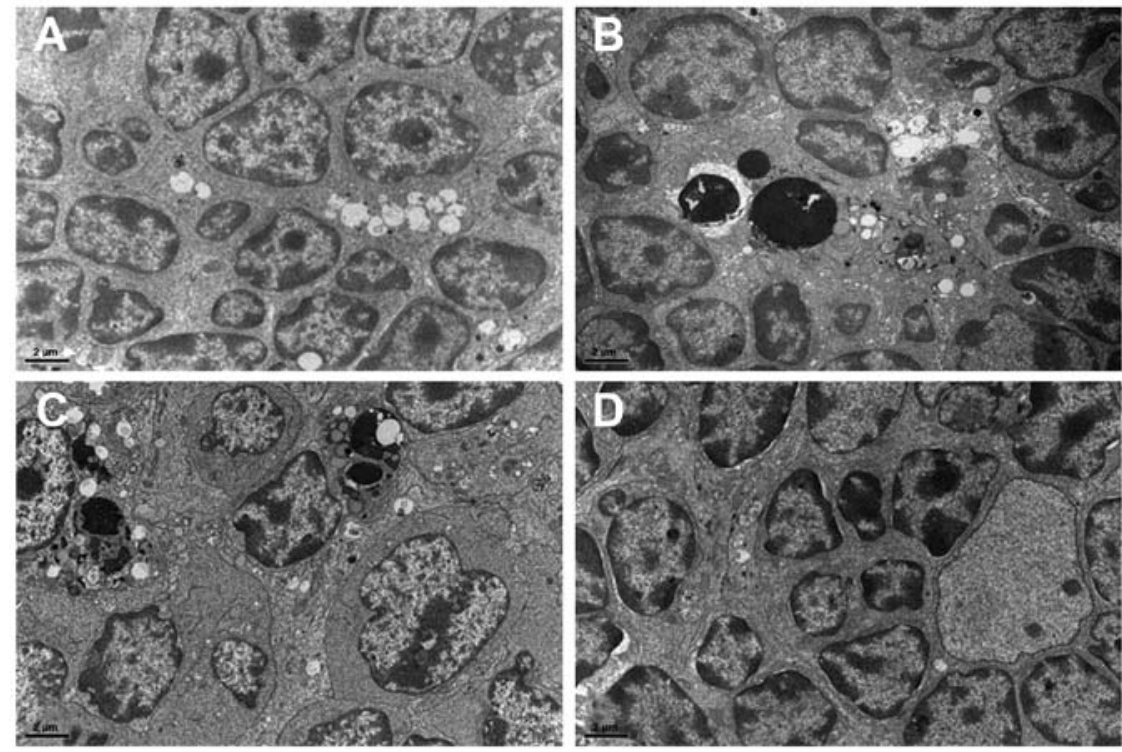

Figure 9. Ultrastructural changes in the mouse thymus $(\mathrm{x} 8,000)$. (A) Electron micrographs of normal thymus. The thymocytes are densely packed in the cortex. A typical thymocyte has a round shape, relatively smooth margin, heterochromatic nucleus and scarce cytoplasm with rare organelles. (B) Electron micrographs of thymocytes in the thymus $12 \mathrm{~h}$ following the administration of recombinant EGFL8 (rEGFL8) protein. Typical apoptotic thymocytes ingested by a macrophage are clearly observed. (C) Electron micrographs of thymocytes in the thymus 1 day following the administration of rEGFL8. The remnants of phagocytized apoptotic thymocytes are observed within the macrophages. (D) Electron micrographs of thymocytes in the involuted thymus 3 days following the administration of rEGFL8.

A

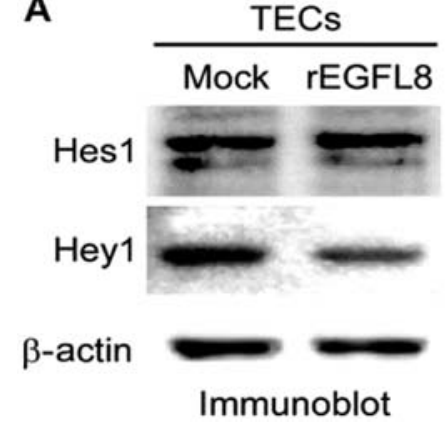

B

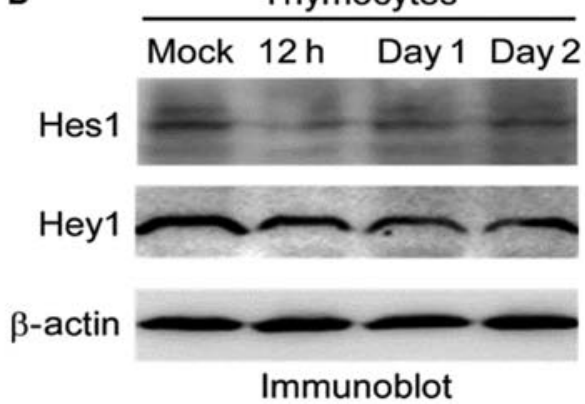

Figure 10. (A) Downregulation of Hes1 and Heyl expression in the mouse thymic epithelial cells (TECs). TECs were incubated with $100 \mathrm{ng} / \mathrm{ml} \mathrm{recom}$ binant EGFL8 (rEGFL8) protein and Hes1 and Hey1 expression was examined $12 \mathrm{~h}$ later by immunoblot assay. $\beta$-actin was used as the loading control. (B) Downregulation of Hes1 and Heyl expression in the mouse thymocytes. Mice were injected intavenously (i.v.) with $100 \mu \mathrm{g}$ rEGFL8 protein and the expression of Hes1 and Heyl was examined at the indicated time points by immunoblot assay. $\beta$-actin was used as the loading control.

rEGFL8. The thymus from the control mice showed normal ultrastructural features (Fig. 9A). However, electron micrographs of the thymus $12 \mathrm{~h}$ after the administration of rEGFL8 revealed apoptotic thymus cells, although their proportion was relatively small (Fig. 9B). Apoptotic cells (Fig. 9B and C) had typical morphological changes; the chromatins were condensed and aggregated into large dark, compact masses. Most of the apoptotic thymic cells appeared to be thymocytes, whereas the apoptosis of TECs and other thymic stromal cell types was rarely observed. In the thymus 1 day after the administration of rEGFL8, apoptotic thymocytes were observed to a much lesser extent than in at $12 \mathrm{~h}$ (Fig. 9C). Within 3 days after the rEGFL8 injection, apoptotic cells were rarely visible and the thymus exhibited an almost normal ultrastructural appearance (Fig. 9D).

Inhibitory effects of rEGFL8 on the expression of HesI and Heyl. To determine the effect of rEGFL8 on the Notch signaling pathway in mouse TECs, TECs were treated with rEGFL8 protein and the expression of Hes1 and Heyl was then assessed by western blot analysis. Remarkably, the expression of Hes1 and Heyl was downregulated in the TECs (Fig. 10A). To determine the effect of EGFL8 on the Notch signaling pathway in mouse thymocytes, rEGFL8 protein was injected into the mice, and the expression of Hes1 and Heyl was assessed by western blot analysis. The expression of Hes1 and Heyl was significantly downregulated in the mouse thymocytes (Fig. 10B). The inhibitory effect of rEGFL8 on Hes1 expression was more pronounced $12 \mathrm{~h}$ after rEGFL8 injection. The decrease in Heyl expression was most evident 1 day following treatment with EGFL8.

\section{Discussion}

In the present study, to our knowledge, we present for the first time an optimized protocol for the production of high-purity mouse rEGFL8 protein; we also demonstrate the biological activity of the EGFL 8 protein in mouse thymocytes. The biological activity of rEGFL8 in mouse thymocytes and TECs 
was investigated as the modulation of the EGFL 8 gene in TECs provided evidence of its negative regulatory role on the activity of TECs and the development of mouse thymocytes in our previous study (13). The in vivo experiment with rEGFL8 demonstrated its inhibitory effect on the development of mouse thymocytes in a time-dependent manner in the present study. rEGFL8 also induced a decrease in the weight of the thymus, as well as in the number of mouse thymocytes primarily by the suppression of thymocyte proliferation as assessed by BrdU cell proliferation assay and, to a lesser extent, by the induction of apoptosis in thymocytes as revealed by flow cytometric analysis and electron microscopy. In a previous study, a differential equation model of thymocyte dynamics was constructed, which showed that cell proliferation, differentiation and cell death in the thymus may account for both the total number of thymic cells and the fraction of various types of immature and mature thymocytes (16). According to this model, a decrease in the proliferation rate or an increase in the rate of apoptosis may be some of the parameters that account for the reduction of thymic size and thymocyte number that occurs due to thymic involution.

As regards the reduction in the number of thymocytes, the suppressive effect of rEGFL8 on thymocytes had no subset specificity, although the administration of rEGFL8 induced a profound decrease in the number of DP thymocytes. The reason why there was a marked reduction in the number of DP thymocytes compared with the other subsets may be related with their proportion and the absolute number in the control mice.

As regards the molecular mechanisms underlying the inhibitory effects of EGFL8 on the proliferation of mouse thymocytes and its promotion of apoptosis in mouse thymocytes, the involvement of EGFL8 in the Notch signaling pathway contributes, at least in part, to this negative regulatory role of rEGFL8, since it inhibited the expression of the Notch downstream effectors, Hes1 and Hey1, in the mouse thymocytes and TECs. Notch signaling is directly involved in the regulation of thymic T-cell development (17). Notch signaling has also been found to play a central role in reconstitution after transplantation, immunomodulation, or the development and maturation of thymocytes (18). Taken together, the data from the present study suggest that EGFL8 acts as a negative regulatory factor in the critical steps of mouse T-cell development, such as thymocyte proliferation and survival, through the inhibition of Notch signaling in mouse thymocytes and TECs.

In conclusion, the pET-28a-EGFL8 vector was expressed in $E$. coli (DE3) and a relatively large amount of mouse rEGFL8 was successfully produced and purified in this study. Treatment with rEGFL8 reduced the weight of the thymus and the number of thymocytes, suppressed thymocyte proliferation, induced thymocyte apoptosis and inhibited Notch signaling (downregulation of Hes1 and Heyl expression) in mouse thymocytes and TECs.

Therefore, the data from the present study suggest that EGFL8 acts as a negative regulatory factor in the critical steps of mouse T-cell development. Further studies are required to fully elucidate the functional role of EGFL8 in diverse physiological and pathological processes.

\section{Acknowledgements}

This study was supported by the National Research Foundation of Korea (NRF) grant funded by the Korean government

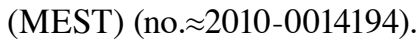

\section{References}

1. Takahama Y: Journey through the thymus: stromal guides for T-cell development and selection. Nat Rev Immunol 6: 127-135, 2006.

2. Fitch MJ, Campagnolo L, Kuhnert F and Stuhlmann H: Egfl7, a novel epidermal growth factor-domain gene expressed in endothelial cells. Dev Dyn 230: 316-324, 2004.

3. Nichol D and Stuhlmann H: EGFL7: a unique angiogenic signaling factor in vascular development and disease. Blood 119: 1345-1352, 2012

4. Campagnolo L, Leahy A, Chitnis S, Koschnick S, Fitch MJ Fallon JT, Loskutoff D, Taubman MB and Stuhlmann H: EGFL7 is a chemoattractant for endothelial cells and is up-regulated in angiogenesis and arterial injury. Am J Pathol 167: 275-284, 2005.

5. Nichol D, Shawber C, Fitch MJ, Bambino K, Sharma A, Kitajewski J and Stuhlmann H: Impaired angiogenesis and altered Notch signaling in mice overexpressing endothelial Egfl7. Blood 116: 6133-6143, 2010.

6. Parker LH, Schmidt M, Jin SW, Gray AM, Beis D, Pham T, Frantz G, Palmieri S, Hillan K, Stainier DY, De Sauvage FJ and Ye W: The endothelial-cell-derived secreted factor Egfl7 regulates vascular tube formation. Nature 428: 754-758, 2004.

7. Schmidt MH, Bicker F, Nikolic I, Meister J, Babuke T, Picuric S, Muller-Esterl W, Plate KH and Dikic I: Epidermal growth factorlike domain 7 (EGFL7) modulates Notch signalling and affects neural stem cell renewal. Nat Cell Biol 11: 873-880. 2009.

8. Durrans A and Stuhlmann H: A role for Egfl7 during endothelial organization in the embryoid body model system. J Angiogenes Res 2: 4, 2010.

9. Chim SM, Qin A, Tickner J, Pavlos N, Davey T, Wang H, Guo Y, Zheng MH and Xu J: EGFL6 promotes endothelial cell migration and angiogenesis through the activation of extracellular signalregulated kinase. J Biol Chem 286: 22035-22046, 2011.

10. Kojika S and Griffin JD: Notch receptors and hematopoiesis. Exp Hematol 29: 1041-1052, 2001

11. Wu F, Shirahata A, Sakuraba K, Kitamura Y, Goto T, Saito M, Ishibashi K, Kigawa G, Nemoto H, Sanada Y and Hibi K: Downregulation of EGFL8: a novel biomarker for advanced gastric cancer. Anticancer Res 31: 3377-3380, 2011.

12. Wu F, Shirahata A, Sakuraba K, Kitamura Y, Goto T, Saito M, Ishibashi K, Kigawa G, Nemoto H, Sanada Y and Hibi K: Downregulation of EGFL8: a novel prognostic biomarker for patients with colorectal cancer. Anticancer Res 31: 2249-2254, 2011.

13. Choi HJ, Yoon TD, Muhammad I, Jeong MH, Lee J, Baek SY, Kim BS and Yoon S: Regulatory role of mouse epidermal growth factor-like protein 8 in thymic epithelial cells. Biochem Biophys Res Commun 425: 250-255, 2012.

14. Romano R, Palamaro L, Fusco A, Iannace L, Maio S, Vigliano I, Giardino G and Pignata C: From murine to human nude/SCID: the thymus, T-cell development and the missing link. Clin Dev Immunol 2012: 467101, 2012.

15. Faas SJ, Rothstein JL, Kreider BL, Rovera G and Knowles BB: Phenotypically diverse mouse thymic stromal cell lines which induce proliferation and differentiation of hematopoietic cells. Eur J Immunol 23: 1201-1214, 1993.

16. Mehr R, Globerson A and Perelson AS: Modeling positive and negative selection and differentiation processes in the thymus. $\mathrm{J}$ Theor Biol 175: 103-126, 1995.

17. Ersvaer E, Hatfield KJ, Reikvam $\mathrm{H}$ and Bruserud O: Future perspectives: therapeutic targeting of notch signalling may become a strategy in patients receiving stem cell transplantation for hematologic malignancies. Bone Marrow Res 2011: 570796, 2011.

18. Pui JC, Allman D, Xu L, DeRocco S, Karnell FG, Bakkour S, Lee JY, Kadesch T, Hardy RR, Aster JC and Pear WS: Notch1 expression in early lymphopoiesis influences B versus T lineage determination. Immunity 11: 299-308, 1999. 